\title{
Europe : SurveyLang, l'enquête européenne sur les compétences langagières
}

\section{Neil Jones}

Traducteur : Robert Elbaz

\section{OpenEdition Journals}

Édition électronique

URL : http://journals.openedition.org/ries/2444

DOI : 10.4000/ries.2444

ISSN : 2261-4265

\section{Éditeur}

Centre international d'études pédagogiques

\section{Édition imprimée}

Date de publication : 1 septembre 2012

Pagination : 18-21

ISBN : 978-2-85420-595-4

ISSN : $1254-4590$

\section{Référence électronique}

Neil Jones, «Europe : SurveyLang, l'enquête européenne sur les compétences langagières », Revue internationale d'éducation de Sèvres [En ligne], 60 | septembre 2012, mis en ligne le 06 février 2015, consulté le 14 novembre 2019. URL : http://journals.openedition.org/ries/2444; DOI : 10.4000/ries. 2444

Ce document a été généré automatiquement le 14 novembre 2019.

(c) Tous droits réservés 


\title{
Europe : SurveyLang, l'enquête européenne sur les compétences langagières
}

\author{
Neil Jones
}

Traduction : Robert Elbaz

1 Les résultats de la première Enquête européenne sur les compétences langagières (ou ESLC ${ }^{1}$ ) ont été publiés ${ }^{2}$ par la Commission européenne le 21 juin 2012.

2 L'ESLC a évalué les compétences en langues étrangères d'élèves du secondaire et a cherché à déterminer les bonnes pratiques d'apprentissage linguistique à partir de questionnaires remplis au niveau national par les élèves, les enseignants et les chefs d'établissement. Les résultats contribueront à définir un indicateur de compétences linguistiques à l'échelle européenne, afin de mesurer les progrès accomplis vers l'objectif fixé par les conclusions du Conseil européen à Barcelone en 2002, qui appelait à mettre en place « une action afin d'améliorer la maîtrise des compétences de base, notamment par l'enseignement d'au moins deux langues étrangères dès le plus jeune âge ».

Quatorze pays européens y ont participé : la Belgique, la Bulgarie, la Croatie, l'Estonie, la France, la Grèce, Malte, les Pays-Bas, la Pologne, le Portugal, la Slovénie, l'Espagne, la Suède et le Royaume-Uni (Angleterre). Les trois communautés linguistiques de Belgique ont participé séparément à l'étude, qui concerne donc en tout seize systèmes éducatifs.

4 Environ cinquante-quatre mille élèves ont été évalués. Chaque pays a fait porter l'étude sur les deux langues les plus largement enseignées parmi l'anglais, le français, l'allemand, l'italien et l'espagnol. Les tests portaient sur la compréhension orale, la compréhension écrite et la production écrite. Il a été jugé qu'évaluer la production orale poserait des problèmes de logistique pour cette première mouture.

5 L'ESLC s'est concentrée sur les niveaux A1 à B2 du Cadre européen commun de référence pour les langues (CECRL) et a publié les résultats par compétences et par niveau du CECRL. 
6 L'ESLC a été menée par SurveyLang, un groupe composé de huit partenaires ayant une expertise en évaluation linguistique, en conception d'enquêtes et de questionnaires, et en psychométrie. Les tests linguistiques français relevaient de la responsabilité du Centre international d'études pédagogiques (CIEP). L'élaboration des tests linguistiques s'est faite dans le cadre d'une collaboration inter-langues rigoureuse afin d'assurer la comparabilité la plus proche entre les cinq langues.

\section{Conclusions liées aux tests linguistiques}

7 Les résultats montrent que le niveau de maîtrise en langues étrangères varie considérablement suivant les pays. Bien que cela confirme largement ce dont on se doutait déjà, l'importance des écarts constatés est sans doute surprenante: la proportion d'élèves atteignant le niveau correspondant à un utilisateur indépendant (B1 ou B2) varie pour l'anglais de $82 \%$ à Malte et en Suède à $14 \%$ en France, et tombe à seulement $9 \%$ en Angleterre en ce qui concerne le français. Pour la seconde langue vivante, le niveau correspondant à un utilisateur indépendant s'élève à $48 \%$ aux PaysBas pour l'allemand, comparé à $4 \%$ en Suède pour l'espagnol ou à $6 \%$ en Pologne pour l'allemand.

8 Nous devrions être prudents dans l'interprétation de ces différences et garder à l'esprit les nombreux facteurs qui rendent une simple comparaison des performances difficile : la classe dans laquelle se trouvent les élèves, leur âge, le nombre d'années d'étude de la langue ainsi que des facteurs culturels externes comme l'exposition à la langue par l'intermédiaire de la télévision, etc.

9 Alors que de nombreux pays atteignent un haut niveau de maitrise, particulièrement en anglais, il existe six pays où au moins $20 \%$ des élèves n'atteignent pas le niveau de l'utilisateur élémentaire (A1) dans au moins une compétence de leur première langue vivante étrangère. Cela est également vrai pour la seconde langue vivante étrangère dans neuf pays, bien qu'il soit important de remarquer que la durée plus brève d'étude peut ici jouer un rôle.

\section{Conclusions liées aux questionnaires}

10 Les questionnaires visent à faciliter la comparaison en matière de politiques linguistiques et de méthodes pédagogiques d'enseignement des langues entre les États membres, en cherchant à identifier et à partager les bonnes pratiques. Ils se concentrent sur les facteurs qui peuvent être modifiés par les politiques éducatives. Les conclusions les plus importantes sont les suivantes.

11 La plupart des élèves commencent l'apprentissage d'une langue étrangère tôt (avant ou pendant l'enseignement primaire) et la grande majorité apprend deux langues étrangères. Selon les pays, il existe toutefois de grandes différences entre les langues apprises, mortes ou vivantes, tant en ce qui concerne la classe à partir de laquelle elles sont enseignées que leur nombre. L'enquête fait apparaître que ces différences sont directement liées au niveau de maîtrise dans la langue étrangère.

12 Les politiques éducatives ont également pour objectif de créer un cadre de vie et d'apprentissage linguistiquement favorable. Selon les pays, nous découvrons des différences nettes entre les opportunités qu'ont les élèves d'apprendre une langue 
informelle. L'étude montre également que d'autres facteurs (perception qu'ont les élèves de la maitrise d'une langue étrangère par leurs parents, exposition des élèves à une langue étrangère et utilisation qu'ils en font, que ce soit au travers des médias traditionnels ou des nouveaux médias) sont associés au niveau de maîtrise atteint dans la langue considérée.

13 L'enquête a fait apparaitre des différences plus réduites entre les pays en matière d'établissements linguistiquement favorables. Seuls les rapports portant sur le volume de langue étrangère parlée durant les cours montrent des différences significatives entre les pays, indiquant que plus une langue est parlée, plus le niveau de maîtrise est élevé. La perception de l'apprentissage de la langue par les élèves est également fortement corrélée avec la maitrise de celle-ci : ceux qui la considèrent comme utile et envisagent son enseignement de façon positive ont les meilleurs résultats, alors que ceux qui trouvent la langue difficile s'en sortent nettement moins bien.

\section{Comment interpréter les conclusions de l'ESLC?}

14 Il est intéressant d'étudier de près la large couverture médiatique que la publication des conclusions de l'ESLC a provoquée dans toute l'Europe. Naturellement, les pays se comparent entre eux et il semble généralement que les résultats confirment l'opinion préconçue qu'ils sont soit "bons " soit "mauvais » en langues. Il se peut qu'il y ait là un fond de vérité si, comme cela semble être le cas, les différences de maîtrise en langues sont bien plus grandes que celles que l'on observe par exemple dans les études portant sur les mathématiques ou les sciences. Les conclusions de cette enquête servent-elles simplement à renforcer la conviction que l'on ne peut remédier à cet état de fait?

15 L'enquête confirme le statut spécial de l'anglais. C'est la première langue vivante la plus largement adoptée ; elle est apprise jusque dans les plus grandes classes ; enfin, elle est perçue comme la plus utile et la plus largement utilisée dans la vie quotidienne. Les motivations pour l'apprendre sont donc grandes. Lorsque la motivation fait défaut comme c'est le cas en Angleterre, où les élèves ne voient souvent aucun intérêt à apprendre une langue étrangère -, la maîtrise linguistique est logiquement insuffisante. Les conclusions de l'ESLC confirment ainsi, entre autres, que la motivation pour apprendre, fondée sur la perception de l'utilité de connaître la langue vivante en question, est capitale pour réussir.

16 Cela crée un dilemme pour la politique linguistique européenne, qui vise à enseigner «la langue maternelle plus deux langues étrangères ». Dans la synthèse analytique qu'elle a faite de l'ESLC, la Commission européenne reconnaît «l'importance de l'anglais comme compétence fondamentale et comme outil pour l'emploi et pour le développement professionnel ». Mais elle note également que « la diversité linguistique demeure d'une importance vitale pour le développement culturel et personnel » et que, " par conséquent, la nécessité d'améliorer les compétences linguistiques pour accroître l'employabilité dans un monde globalisé doit être associée à la promotion de la diversité linguistique et du dialogue interculturel. »

17 Si l'on se projette dans l'avenir, il s'agit sans doute là du véritable défi auquel est confronté l'enseignement multilingue en Europe. Mon opinion est que l'opposition entre utilité et diversité n'a pas lieu d'être. La véritable utilité de savoir parler une langue étrangère repose précisément sur la connaissance interculturelle et sur la 
capacité que cette aptitude crée de communiquer à un niveau dépassant largement celui de la simple transaction, niveau que l'on ne peut atteindre qu'en parlant la langue de son interlocuteur. Ce qui revient à dire que l'anglais ne jouit en réalité d'aucun privilège particulier.

L'enquête de l'ESLC apporte des preuves qui confirment que le fait d'utiliser une langue dans une optique de communication favorise l'apprentissage. De mon point de vue, la politique linguistique la plus adaptée à l'Europe du XXI siècle sera celle qui accordera une place centrale à la communication et aux compétences interculturelles.

\section{NOTES}

1. ESLC: European Survey on Language Competences.

2. Le rapport final de l'ESLC est consultable sur : http://ec.europa.ec/languages/eslc/docs/en/.

\section{INDEX}

Index géographique : Europe, Belgique, Bulgarie, Croatie, Estonie, France, Grèce, Malte, PaysBas, Pologne, Portugal, Slovénie, Espagne, Suède, Angleterre

Mots-clés : analyse comparative, enseignement des langues, test de langue, apprentissage d'une langue étrangère, compétence linguistique, compétence langagière, enquête

\section{AUTEURS}

\section{NEIL JONES}

Neil Jones a enseigné l'anglais dans différents pays, y compris en Pologne et au Japon, où il a créé des départements et des programmes d'enseignement au niveau universitaire. Titulaire d'un doctorat sur l'utilisation de la théorie des réponses à des items, il a rejoint l'équipe de certification Cambridge ESOL (anglais langue étrangère) en 1993, contribuant à la mise en place de bases de données d'items et de systèmes de tests adaptés à l'ordinateur. Il a également travaillé pour différents cadres d'évaluation multilingues, comme ALTE, CEFR et le plan Asset Languages mis au point pour les écoles britanniques, dont il était le directeur de recherche. Il dirige SurveyLang, la première Étude européenne sur les compétences linguistiques, un projet coordonné par Cambridge ESOL. 